\title{
Linking Soil Chemical Parameters and Fungal Diversity in Qatar
}

Sakeenah Adenana, Jane Ojac, Talaat Abdel-Fattahc', Juha Alataloac, Amjad Shraimb', Mohammed Alsafran ${ }^{\mathrm{d}}$

aDepartment of Biological \& Environmental Science, College of Arts \& Science, Qatar University

bDepartment of Chemistry \& Earth Science, College of Arts \& Science, Qatar University

'Environmental Science Centre, Qatar University

dCentral Lab Unit, Qatar University

\section{Introduction}

Given the vast expanse of Qatar's dryland ecosystems, agricultural productivity and soil stability is highly dependent on the diversity of soil microbiota. The soil environment is a heterogeneous habitat shaped by various components like chemical (organic matter, salinity and nutrients) and biological (microbial diversity, abundance and vegetation) properties that form multitudes of different microhabitats. Soil microbial diversity changes along environmental gradients and it is hypothesized that a "stable" microhabitat is one that is inhabited by a large diversity of established microorganisms that are best adapted to the niche. Microorganisms like fungi serve as the underlying biological drivers for biochemical processes within the soil.

\section{Research Objectives}

The key objective of this study is to evaluate the fungal diversity and abundance present within the Qatari soil using molecular-based tools. Subsequently, this study also aims to evaluate potential relationships between the identified fungal communities with chemical properties of the habitat.

\section{Methodology}

1. Sample Collection and Processing

Soil samples were collected from 19 locations across Qatar, all of which differed in habitat and vegetation types. 20 individual samples were collected approximately $8 \mathrm{~m}$ away from the core vegetative spot. Composite soil samples for each sampled location were then prepared by pooling all 20 core samples; and consequently manually grinded and homogenized.

\section{Study Sites}

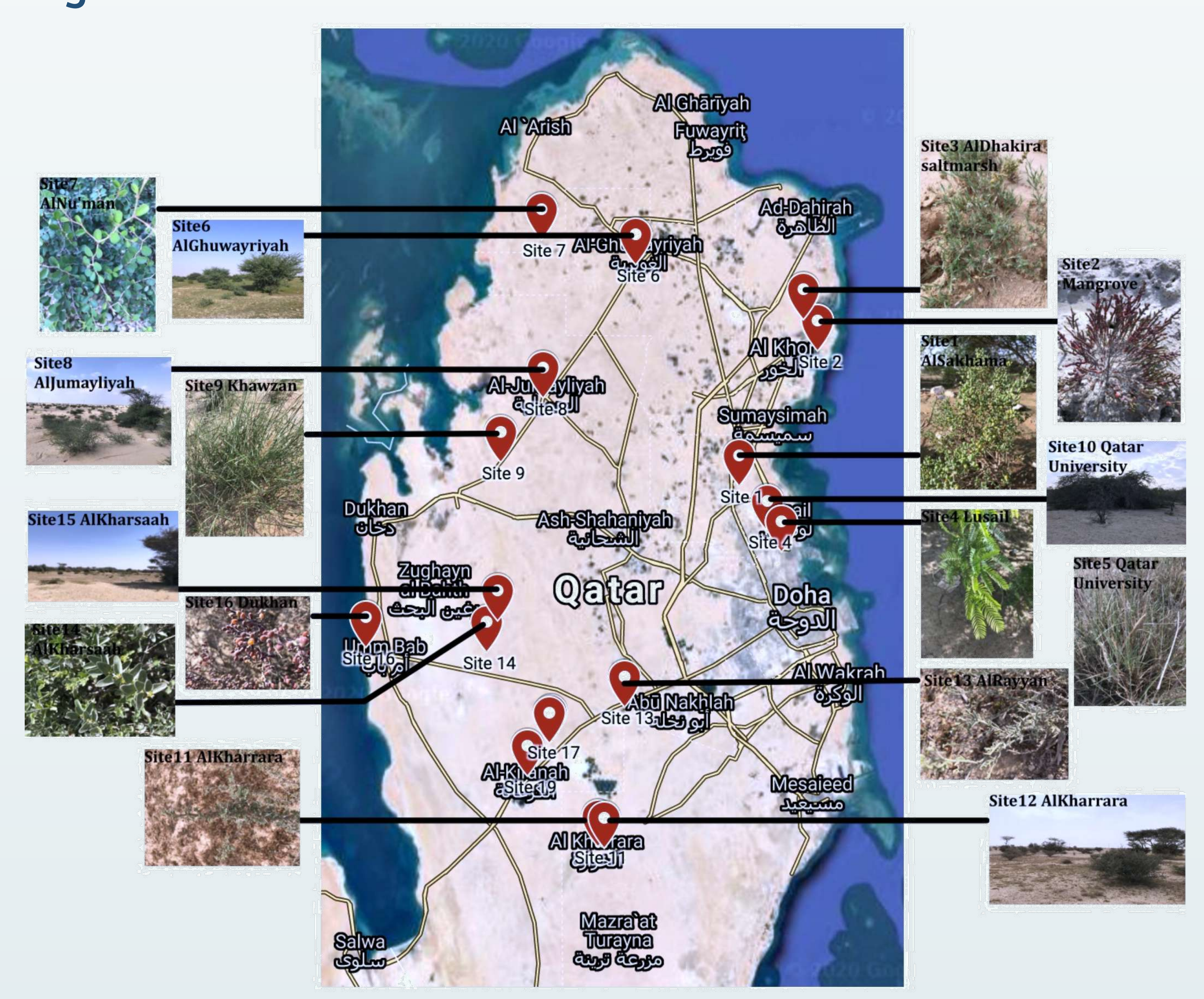

2. Chemical Analysis

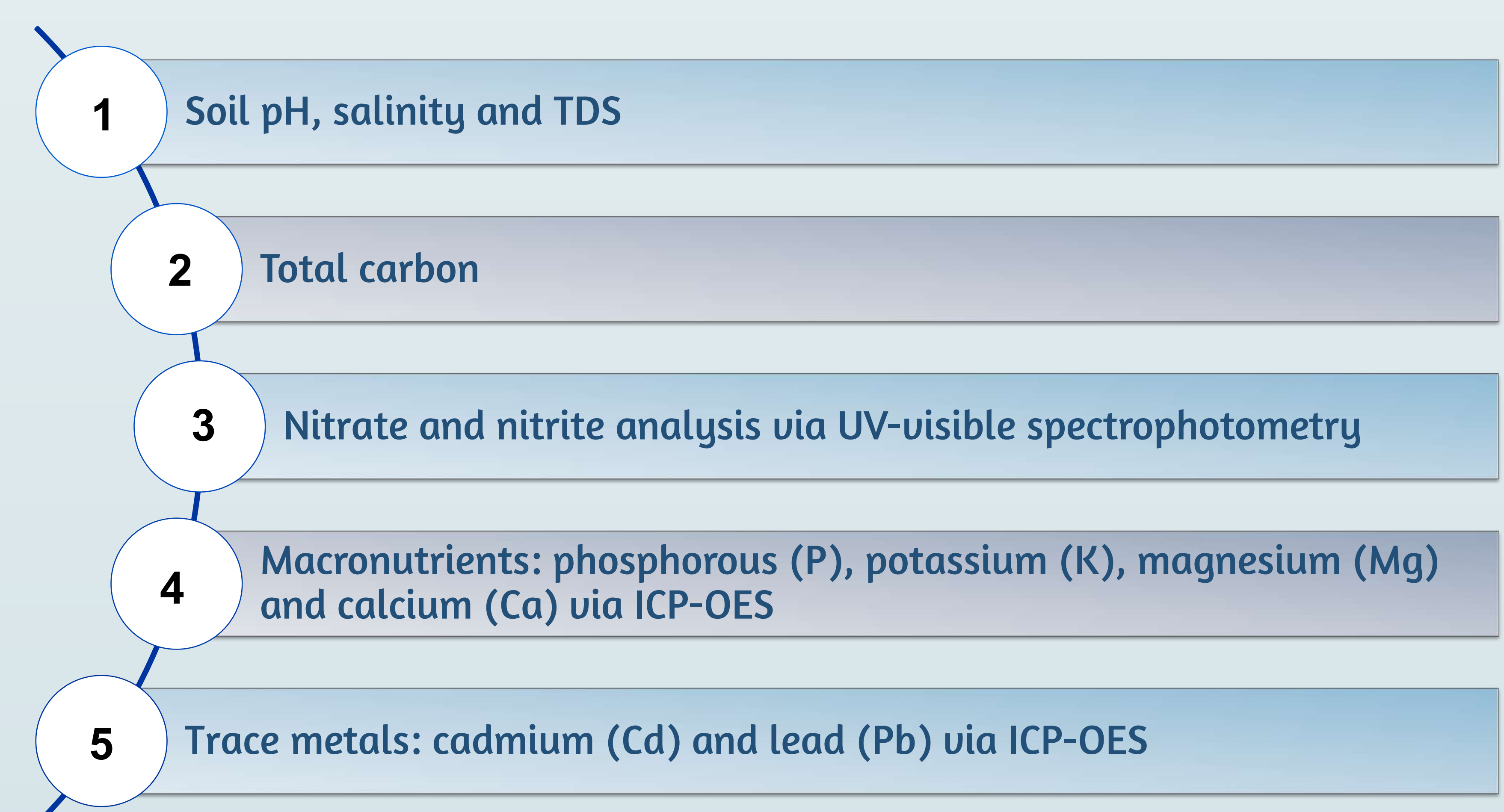

\section{Molecular Analysis}

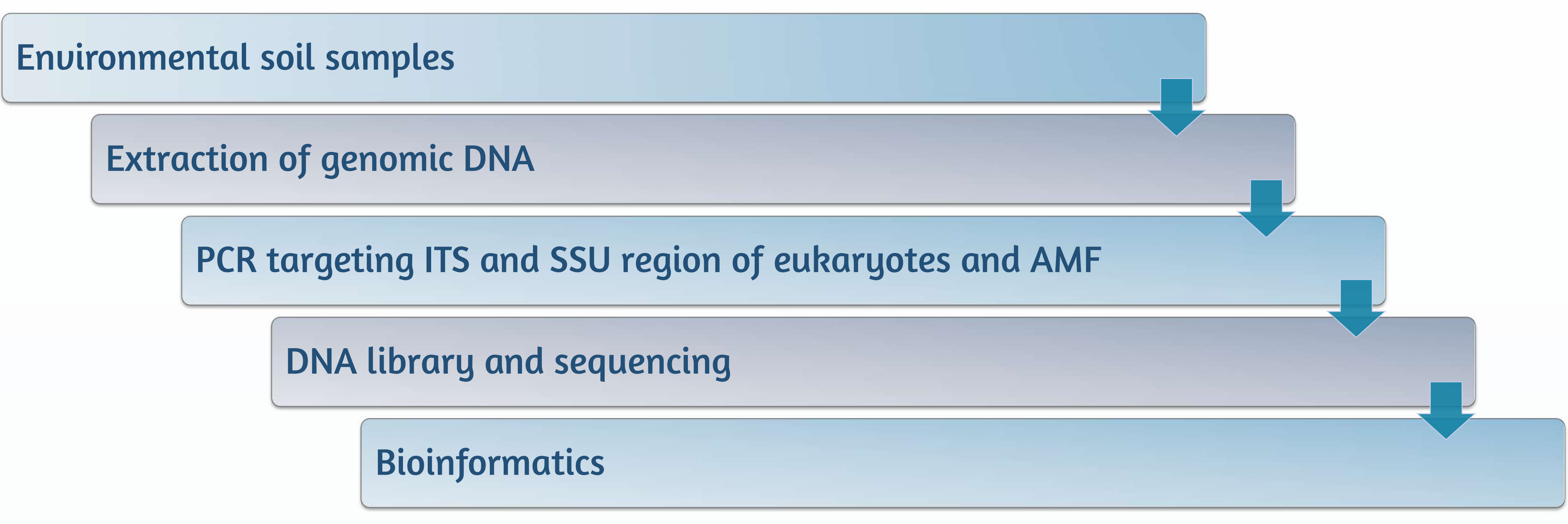

Fig. 1 1.5\% gel electrophoresis images of amplified genomic DNA targeting (a) the internal transcribed spacer (ITS) region of eukaryotes using tagged forward and reverse primers ITS9mun and ITS4ngsUni, (b) a portion of the small subunit (SSU) gene using tagged forward and reverse primers Wanda and AML2 that target AMF sequences

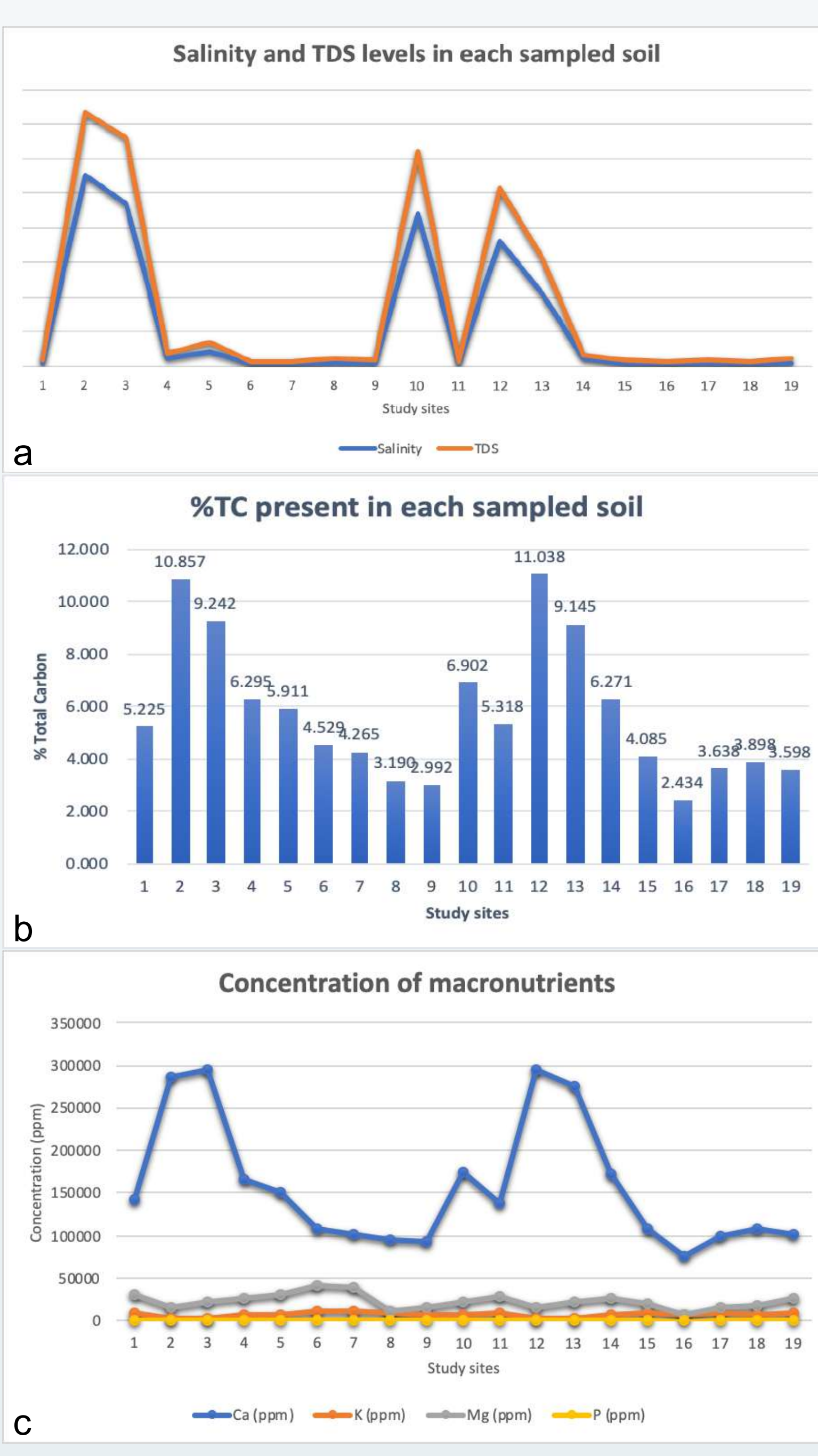

Fig. 2 Trend in chemical parameters measured in each sampled soil

\begin{abstract}
The composition of fungi and AMF varies between different habitats around Qatar. We show that soil fungal communities differs according to the biological (i.e. vegetation type) and chemical (i.e. salinity, TC and nutrients) properties of the soil environment. These findings are consistent with evidence that have shown that vegetation types can influence soil microbial communities.

Despite the lack of significant differences in the measured soil chemical parameters, AMF species are more abundant than that of other fungal species in most of the study sites. However, the high levels of salinity seen in sites 2 and 3 does not directly influence fungal richness as some areas with low salinity also indicated little to no fungal DNA. Although chemical properties are important factors that shape soil communities, other factors like land use may also be an essential component explaining the variation in fungal communities.
\end{abstract}

\section{Future Applications}

This culture-independent method bypasses the need to isolate and culture individual species and allows us to study the function and diversity of fungi, while finding genes with desired biological activity.

\section{Preliminary Results \& Discussion

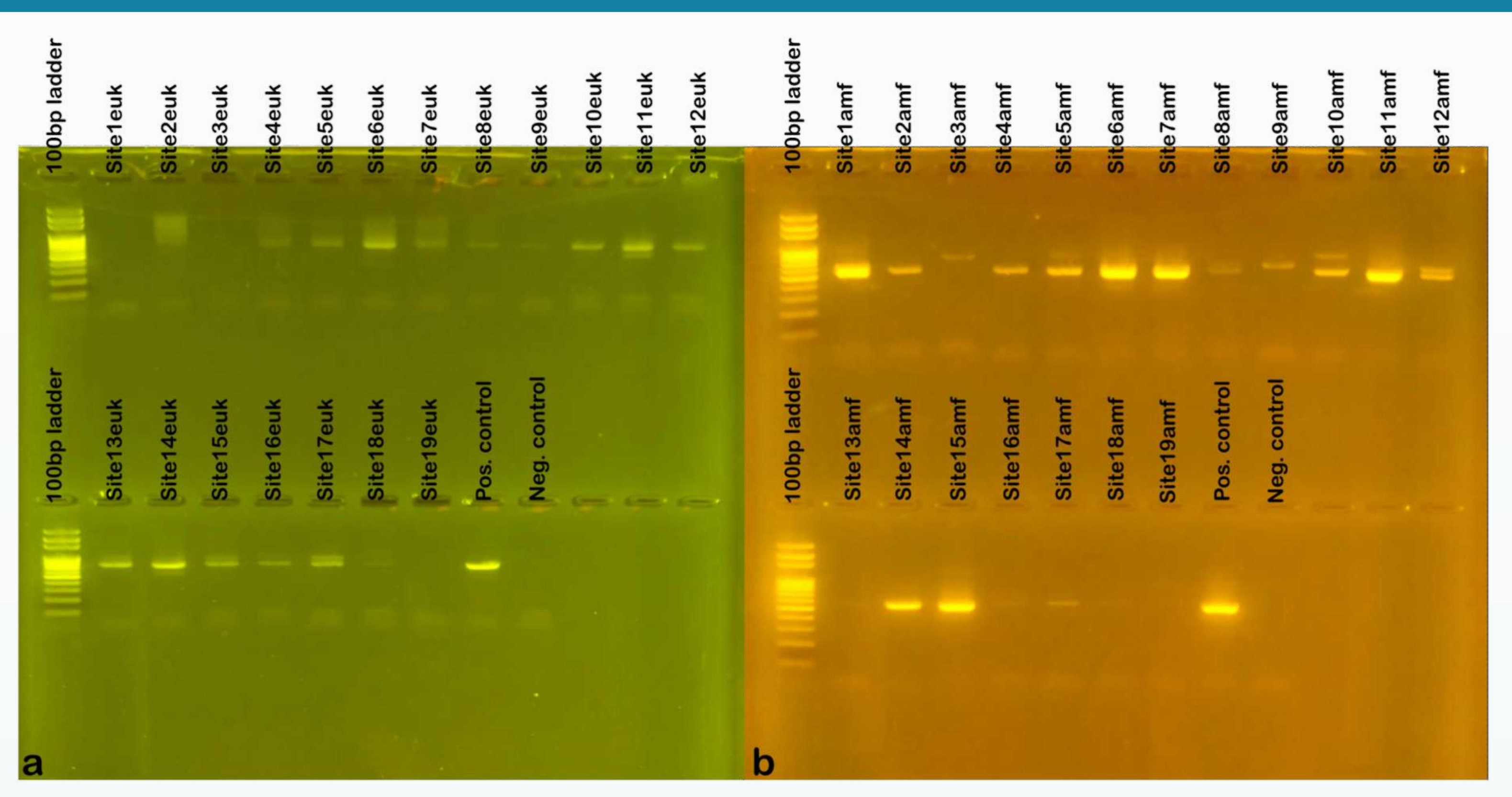
Sustainability Sustainability 